\title{
AT1/2R affects the proliferation and apoptosis of chondrocytes induced by oxygen-glucose deprivation
}

\author{
Cai $\mathrm{HQ}^{1}$, Miao $\mathrm{MY}^{1}$, Zhang $\mathrm{WL}^{1}$ \\ Department of Orthopaedics, Shanghai Children's Medical Center, Shanghai Jiaotong University School of Medicine, \\ Shanghai, China. wlzhangzwl@126.com
}

\begin{abstract}
AIM: Investigate how AT1/2R affected the proliferation and apoptosis of chondrocytes induced by oxygenglucose deprivation.

METHODS: The proliferation and apoptosis of ATDC5 cells was detected by CCK-8 assay and flow cytometry analysis. The expression of Bax, Bcl-2, caspase-3, cleaved-caspase-3, AT1R, AT2R and HIF-1 was determined by Western blot analysis. The collagen II expression was detected by ELISA assay.

RESULTS: Increased ratio of AT1R to AT2R induced by Ang II suppressed the proliferation of oxygen-glucose deprivation ATDC5 cells. Telmisartan, as AT1R inhibitor, promoted the proliferation and inhibited the apoptosis of ATDC5 cells and oxygen-glucose deprivation ATDC5 cells. The collagen II expression either intracellular or cellular supernatant was decreased after Ang II treatment, which was reversed by telmisartan. And, telmisartan reduced the AT1R expression while increased the AT2R expression in ATDC5 cells and oxygenglucose deprivation ATDC5 cells.

CONCLUSIONS: Ang II caused an increased ratio of AT1R to AT2R, which suppressed the proliferation of oxygen-glucose deprivation ATDC5 cells. Furthermore, telmisartan caused a decrease of AT1R and increase of AT2R, which promoted the proliferation and inhibited the apoptosis of oxygen-glucose deprivation ATDC5 cells. This new finding could provide a new insight into the treatment of osteoarthritis (Fig. 4, Ref. 19). Text in PDF www.elis.sk

KEY WORDS: angiotensin II type 1/2 receptor, chondrocytes, proliferation, apoptosis, oxygen-glucose deprivation.
\end{abstract}

\section{Introduction}

Osteoarthritis (OA) is a chronic bone and joint disease caused by articular cartilage degeneration and hyperosteogeny and gonarthrosis are most common. Knee osteoarthritis (KOA) is characterized by articular cartilage degeneration, osteoporosis and synovitis (1-3). With the increasing incidence of OA, it has become an increasingly concerning social problem. At present, there is no effective radical cure for OA, which mainly focuses on the control of symptoms and improvement of joint function.

Studies indicated that OA was correlated with hypertension (4-6). Renin-angiotensin system (RAS) plays a key role in the regulation of cardiovascular system. RAS regulates the blood

'Department of Orthopaedics, Shanghai Children's Medical Center, Shanghai Jiaotong University School of Medicine, Shanghai, China H.Q. Cai and M.Y. Miao contributed equally.

Address for correspondence: W.L. Zhang, Department of Orthopaedics, Shanghai Children's Medical Center, Shanghai Jiaotong University School of Medicine, No. 1678 Dongfang Road, Shanghai 200127, P.R. China.

Acknowledgement: Medium-and Long-Term Clinical Research Foundation of Shanghai Children's Medical Center (Grant No. ZCQSCMC2018-1), the Clinical Research Cultivation Foundation of the Shanghai Shenkang Hospital Development Center (Grant No. SHDC12018X31), the National Natural Science Foundation of China (Grant No. 81801919) pressure and fluid balance to maintain homeostasis. Angiotensin II (Ang II) is an important effective factor in RAS system and Ang II combines with Angiotensin II type 1/2 receptor (AT1/2R) to exert the corresponding physiological effects. The abnormal increase of AT1R activity has an important relationship with vascular remodeling, diabetic nephropathy, atherosclerosis and inflammation (7). However, stimulation of AT2R has physiological effects opposite to stimulation of AT1R, such as vasodilation, inhibition of inflammatory response, anti-fibrosis, and promotion of neuronal repair and regeneration (8-11). Study showed that Ang II, AT1R, AT2R iNOS and TGM2 were significantly increased in rheumatoid arthritis (RA), but AT1R was more significantly increased than AT2R (12). The ratio of AT1R to AT2R was significantly increased which has broken the original balance of AT1R/AT2R. In OA, increased expression of Ang II, AT1R and AT2R also exists (13-15). Therefore, we speculated that the changed expression of Ang II, AT1R and AT2R may lead to occurrence of OA.

The hypoxic environment is also closely related to OA, and the effect and mechanism of anoxia on OA have been discovered and confirmed by continuous research (16). However, there is still no direct evidence about above mechanisms for the effect of anoxia on chondrocytes. Therefore, this study was aimed to investigate how AT1/2R expression affected proliferation and apoptosis of chondrocytes induced by oxygen-glucose deprivation. 


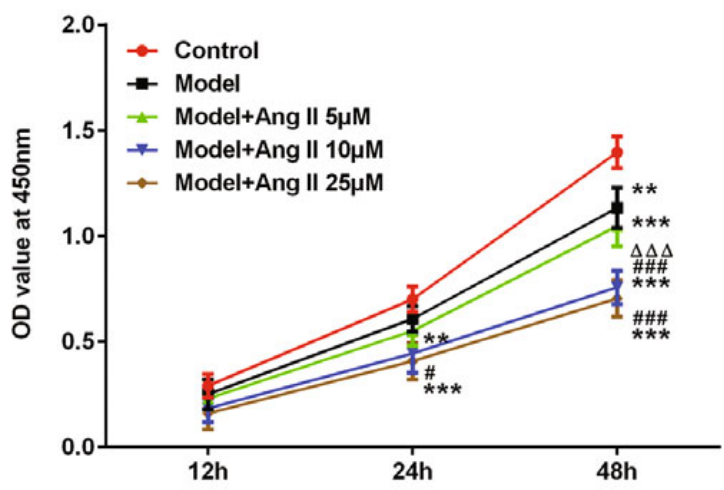

Fig. 1. Ang II affects the proliferation of oxygen-glucose deprivation chondrocytes. $* * \mathbf{p}<0.01$ and $* * * \mathbf{p}<0.001$ vs control group. ${ }^{\# \#} \mathbf{p}$ $<0.001$ vs model group. ${ }^{\Delta \Delta \Delta} \mathrm{p}<0.001$ vs model+Ang II $5 \boldsymbol{\mu M}$ group.

\section{Materials and methods}

Model of oxygen-glucose deprivation chondrocytes.

Chondrocytes (ATDC5 cells) were purchased from Nanjing Cobioer Biosciences Co., Ltd. ATDC5 cells were cultured in DMEM/ F12 medium (containing $10 \%$ fetal bovine serum and $1 \%$ penicillinstreptomycin). When cell fusion reached $90 \%$, chondrocyte inducing conditional media was added for further incubation. All cells were placed in a $37^{\circ} \mathrm{C}$ and $5 \% \mathrm{CO}_{2}$ incubator for culture. ATDC5 cells were divided into control group, model group and model+Ang II group. In the model group, the serum-containing medium was removed by washing the cells with PBS for three times. Then, ATDC5 cells were treated with sugar-deficient solution and incubated in $95 \% \mathrm{~N}_{2}$ and $5 \% \mathrm{CO}_{2}$ incubator for $6 \mathrm{~h}$. In model+Ang II groups, oxygen-glucose deprivation ATDC 5 cells were separately treated with 5, 10 and $25 \mu \mathrm{M}$ Ang II and $24 \mathrm{~h}$ later, 5, 10 and $25 \mu \mathrm{M}$ Ang II was separately added to cells for incubation for another $48 \mathrm{~h}$.

\section{Telmisartan treatment.}

The oxygen-glucose deprivation ATDC5 cells were treated with $10 \mu \mathrm{M}$ telmisartan (Telm) for $1 \mathrm{~h}$ and $10 \mu \mathrm{M}$ Ang II for $24 \mathrm{~h}$. Then, $10 \mu \mathrm{M}$ Ang II was added to cells for incubation of another $48 \mathrm{~h}$.
CCK-8 assay.

ATDC5 cells $\left(1 \times 10^{4} /\right.$ well $)$ were inoculated in 96-well plates. After cells were treated with different concentrations of Ang II, 10 $\mu \mathrm{L}$ CCK8 solution was added to each well, and the culture plate was incubated in an incubator for $3 \mathrm{~h}$. The absorbance (OD) value at $450 \mathrm{~nm}$ was determined by a microplate reader.

\section{Western blot analysis.}

ATDC5 cells in different treatment groups were added with RIPA lysate to extract the proteins. The concentration of extracted proteins was determined according to the instructions of the BCA protein detection kit. The protein samples of the same concentration were subjected to SDS-PAGE electrophoresis. The membrane was treated with $5 \%$ non-fat milk powder for $2 \mathrm{~h}$. The primary antibodies (anti-Bax, anti-Bcl-2, anti-caspase-3, anti-cleaved-caspase-3, anti-AT1R, anti-AT2R and anti-HIF-1) were added to the membrane which was blocked overnight at $4{ }^{\circ} \mathrm{C}$. After washing with TBST, a HRP-coupled secondary antibody was added to the membrane which was incubated at room temperature for $1 \mathrm{~h}$. The protein density was quantitatively analyzed by Image $J$ software and GAPDH was used as an internal control. The expression level of the target protein was reflected by the ratio of the gray value of the target protein band to GAPDH band.

\section{Flow cytometry analysis.}

ATDC5 cells in different treatment groups were collected and centrifuged at $1000 \mathrm{~g}$ for $5 \mathrm{~min}$. After the supernatant was discarded, $195 \mu \mathrm{L}$ Annexin v-FITC solution was added to suspend the cells and then $5 \mu \mathrm{L}$ Annexin v-FITC solution was added and the cells were mixed gently. $10 \mu \mathrm{L}$ propidium iodide (PI) was added to mixed cells, which was incubated at room temperature in dark for $15 \mathrm{~min}$. The sample was placed on FACSCalibur flow cytometer to detect cell apoptosis.

\section{ELISA assay.}

The ATDC5 cells in control group, control+Ang II group and control+Ang II+Telm group were selected and centrifuged to separate the cells and supernatant. The level of Collagen II in cells and supernatant was detected by ELISA assay according to the kit instructions.
A

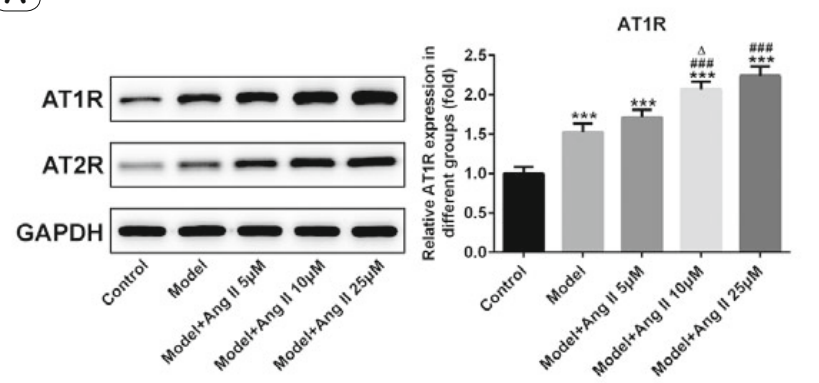

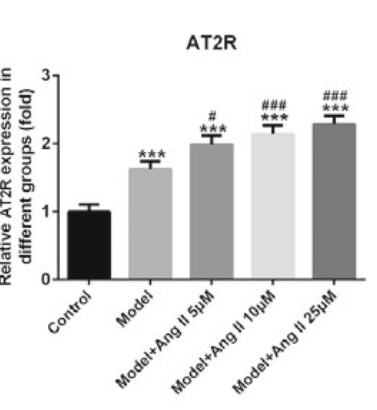

(B)

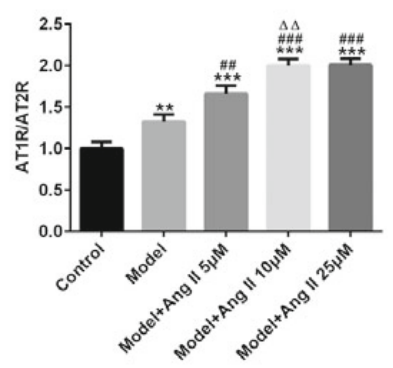

Fig. 2. Ang II affects the expression of AT1/2R. (A) The expression of AT1R and AT2R in ATDC5 cells and oxygen-glucose deprivation ATDC5 cells was detected by Western blot analysis. ${ }^{* * *} \mathbf{p}<0.001$ vs Control group. ${ }^{\# \#} \mathbf{p}<0.001$ vs model group. ${ }^{\Delta} \mathbf{p}<0.05$ vs model + Ang II $5 \mu$ M group. (B) The ratio of AT1R to AT2R in ATDC5 cells and oxygen-glucose deprivation ATDC5 cells. ** p $<0.01$ and $* * * p<0.001$ vs control group. ${ }^{\#}$ $\mathrm{p}<0.01$ and ${ }^{\#} \mathrm{p}<\mathbf{0 . 0 0 1}$ vs model group. ${ }^{\Delta \Delta} \mathrm{p}<\mathbf{0 . 0 1}$ vs model+Ang II $5 \boldsymbol{\mu M}$ group. 
$584-588$

A

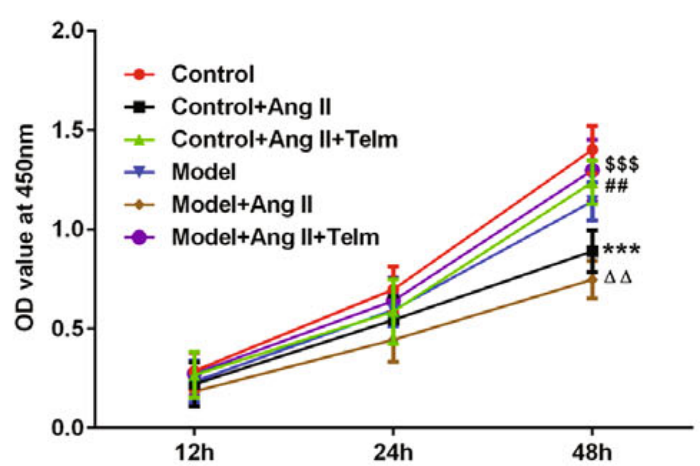

B
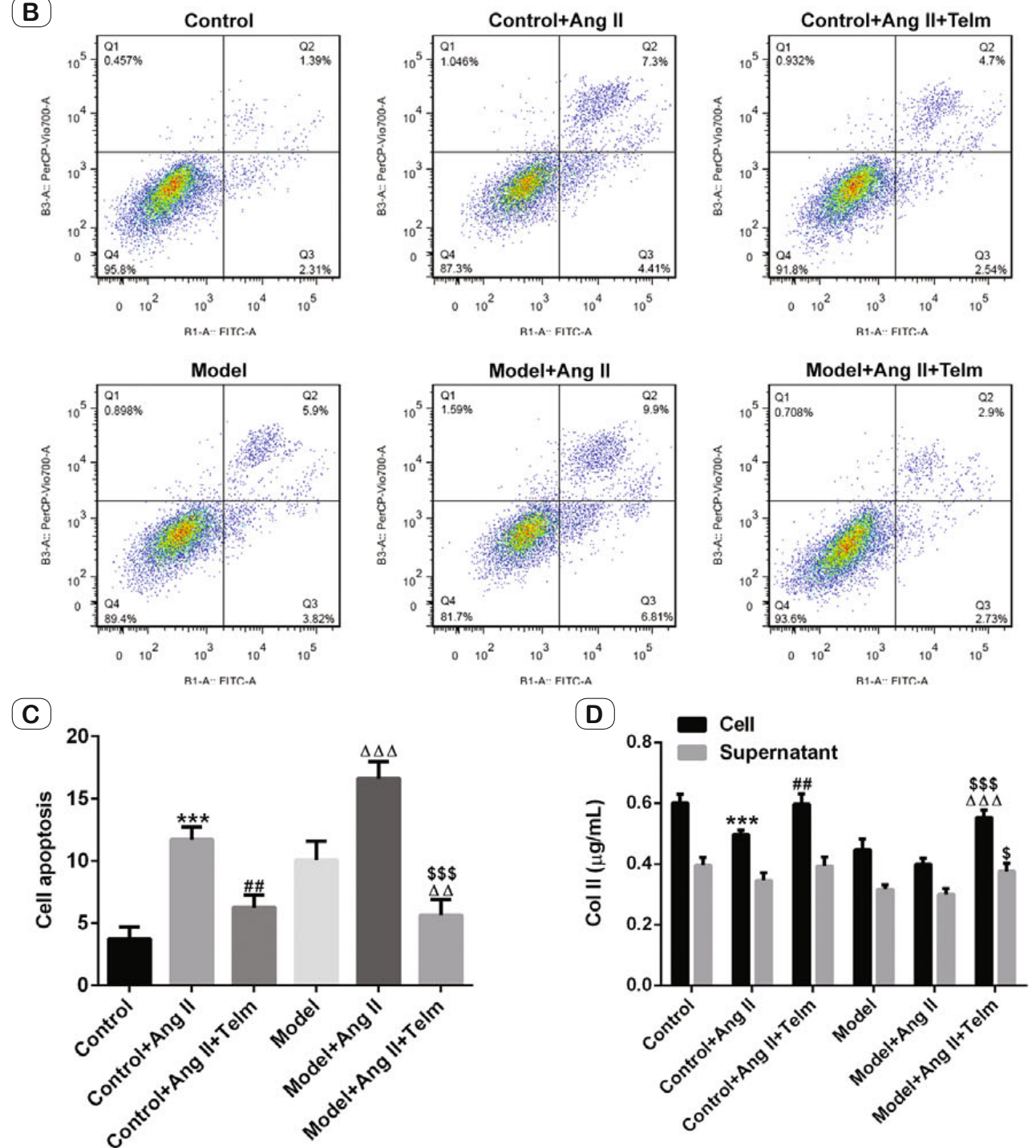

Fig. 3. Telmisartan affects the proliferation, apoptosis and collagen II expression of oxygen-glucose deprivation chondrocytes. (A) The proliferation of ATDC 5 cells and oxygen-glucose deprivation ATDC 5 cells was detected by CCK-8 assay after treatment with telmisartan (Telm). *** $p<$ 0.001 vs control group. ${ }^{\#} p<0.01$ vs Control+Ang II group. ${ }^{\Delta \Delta} p<0.01$ vs model group. ${ }^{\text {sss }} p<0.001$ vs model+Ang II group. (B/C) The apoptosis of ATDC5 cells and oxygen-glucose deprivation ATDC5 cells was detected by flow cytometry analysis after treatment of telmisartan (Telm). $* * * p<0.001$ vs control group. ${ }^{\#} p<0.01$ vs control+Ang II group. ${ }^{\Delta \Delta} p<0.01$ and ${ }^{\Delta \Delta \Delta} p<0.001$ vs model group. ${ }^{\text {sss }}$ p $<0.001$ vs model+Ang II group. (D) The collagen II expression in ATDC5 cells and oxygen-glucose deprivation ATDC5 cells and supernatant after treatment of telmis$\operatorname{artan}\left(\right.$ Telm) was analyzed by ELISA assay. ${ }^{* * *} \mathbf{p}<0.001$ vs control group. ${ }^{\# \#} \mathbf{p}<0.01$ vs control+Ang II group. ${ }^{\Delta \Delta \Delta}$ p $<0.001$ vs model group. ${ }^{s} p<0.05$ and ${ }^{\text {sss }} p<0.001$ vs model+Ang II group. 
Statistical analysis.

SPSS 19.0 software was used for statistical analysis of all the data. Measurement data were expressed as mean \pm standard deviation. One-way analysis of variance was used for comparison among multiple groups, and LSD-t method was used for multiple comparisons among groups. $\mathrm{p}<0.05$ was considered statistically significant.

\section{Results}

Ang II affects the proliferation of oxygen-glucose deprivation chondrocytes

After ATDC5 cells were treated with sugar-deficient solution and incubated in $95 \% \mathrm{~N}_{2}$ and $5 \% \mathrm{CO}_{2}$ incubator, the proliferation activity was gradually decreased form $12 \mathrm{~h}$ to $48 \mathrm{~h}$. Moreover, Ang II treatment further inhibited the cell proliferation activity. The inhibition effect of $25 \mu \mathrm{M}$ Ang II treatment on cell proliferation activity was not obvious with that of $10 \mu \mathrm{M}$ Ang II treatment (Fig. 1).

\section{Ang II affects the expression of AT1/2R}

The expression of AT1/2R was increased in oxygen-glucose deprivation ATDC5 cells (Fig. 2A). Furthermore, Ang II treatment promoted the expression of AT $1 / 2 \mathrm{R}$ in oxygen-glucose deprivation
ATDC 5 cells and the promotion effect of $25 \mu \mathrm{MAng}$ II treatment was not obvious with that of $10 \mu \mathrm{M}$ Ang II treatment. As shown in Figure $2 \mathrm{~B}$, the ratio of AT1R to AT2R was increased and Ang II treatment further up-regulated the ratio. The effect of $25 \mu \mathrm{M}$ Ang II treatment on ATDC5 cells was not obvious with $10 \mu \mathrm{M}$ Ang II treatment.

Telmisartan affects the proliferation, apoptosis and collagen II expression of oxygen-glucose deprivation chondrocytes

As shown in Figure 3A, Ang II treatment suppressed the proliferation activity of ATDC5 cells and oxygen-glucose deprivation ATDC 5 cells and telmisartan could improve the proliferation activity of those cells. As shown in Figures 3B and 3C, the cell apoptosis was enhanced either in control+Ang II group or model+Ang II group while telmisartan decreased apoptosis of ATDC5 cells and oxygen-glucose deprivation ATDC5 cells. The collagen II expression in Ang II treatment group was decreased while telmisartan improved the collagen II expression in either intracellular or cellular supernatant (Fig. 3D).

Telmisartan affects the expression of apoptosis-related proteins, AT1/2R and HIF-1

The expression of Bax, c-casp-3 and HIF-1 was up-regulated and $\mathrm{Bcl}-2$ expression was down-regulated either in control+Ang
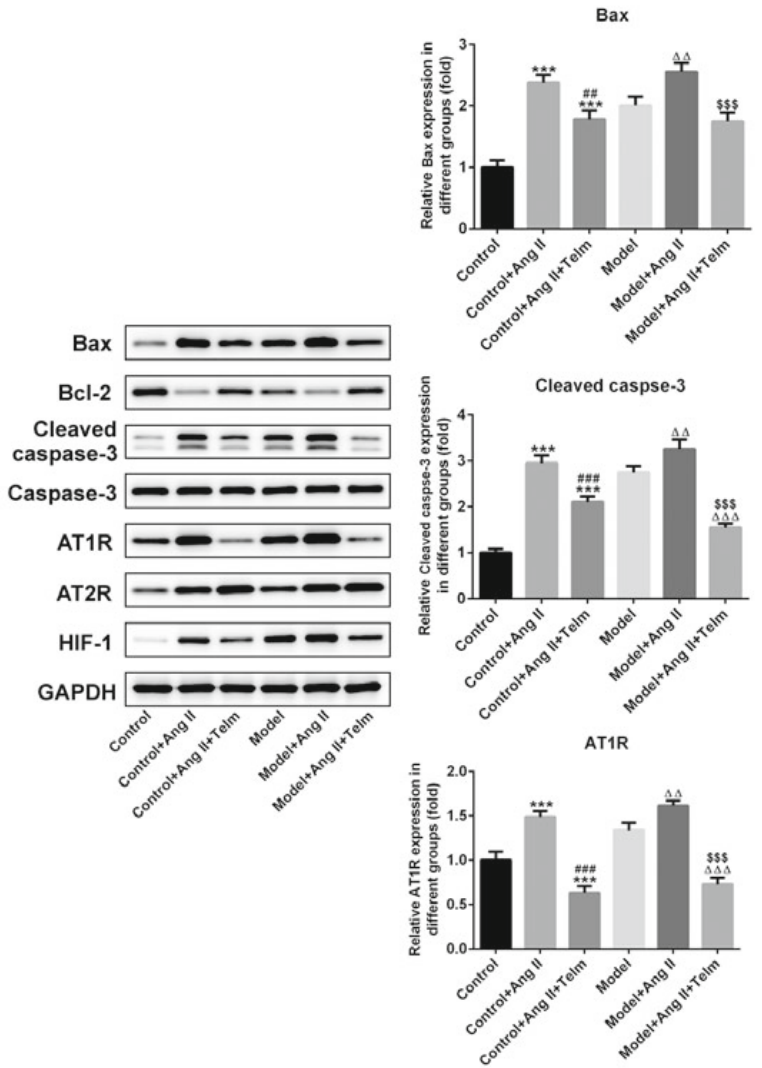
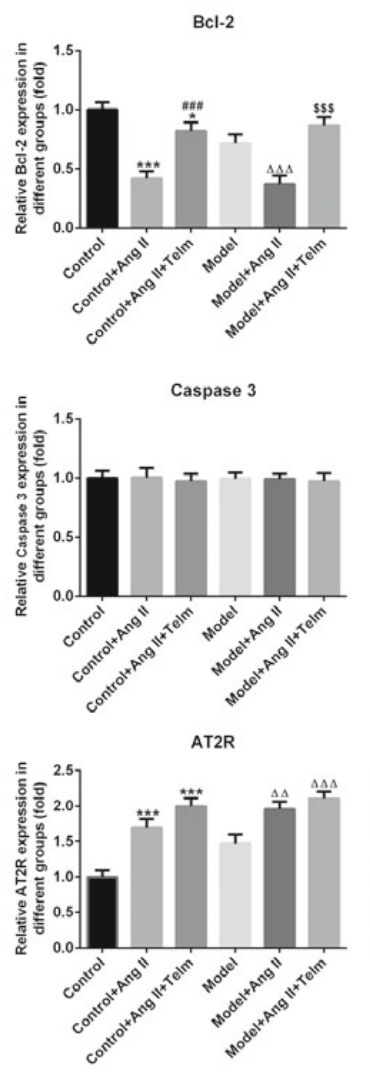

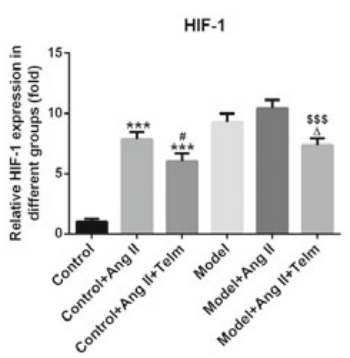

Fig. 4. Telmisartan affects the expression of apoptosis-related proteins, AT1/2R and HIF-1. The expression of Bax, Bcl-2, Cleaved-caspase-3, Caspase-3, AT1R, AT2R and HIF-1 in ATDC5 cells and oxygen-glucose deprivation ATDC5 cells fter treatment of telmisartan (Telm). * $p<$ 0.05 and ${ }^{* * *} p<0.001$ vs control group. ${ }^{\#} p<0.05,{ }^{\# \#} p<0.01$ and ${ }^{\# \# \#} p<0.001$ vs control+Ang II group. ${ }^{\Delta} p<0.05,{ }^{\Delta \Delta} p<0.01$ and ${ }^{\Delta \Delta \Delta} p<0.001$ vs model group. ${ }^{\mathrm{ss}} \mathrm{p}<\mathbf{0 . 0 0 1}$ vs model+Ang II group. 
II group or model+Ang II group. Ang II treatment promoted the AT1/2R expression in ATDC5 cells and oxygen-glucose deprivation ATDC 5 cells. And, telmisartan reduced the AT1R expression while further increased the AT2R expression in ATDC5 cells and oxygen-glucose deprivation ATDC5 cells (Fig. 4).

\section{Discussion}

Here, how AT1/2R expression affected the chondrocytes disintegration induced by oxygen-glucose deprivation was explored. It was shown that increased ratio of AT1R to AT2R suppressed the proliferation of oxygen-glucose deprivation ATDC5 cells which was promoted by the decreased ratio of AT1R to AT2R.

Ang II has two receptor subtypes called AT1R and AT2R, and they were considered to be involved in the progression of RA (17 18). Studies indicated that expression of Ang II, AT1R and AT2R was increased in OA (13-15). In this study, model of oxygenglucose deprivation chondrocytes simulating the OA model in vitro was constructed. The expression of AT1R and AT2R was also increased in oxygen-glucose deprivation chondrocytes and ratio of AT1R to AT2R was also increased.

AT2R expression was increased in spleen and synovial tissue of adjuvant arthritis (AA) rats and intra-articular injection of AT2R agonist could reduce the infiltration of macrophages and Tlymphocytes in synovium to inhibit the abnormal proliferation of synoviocytes. Stimulation of AT2R could antagonize the high activity and strong chemotactic ability of peripheral monocytes mediated by IL-1 and inhibition of AT1R signal transduction played a protective role in the course of RA. The imbalance of AT1R/AT2R may be involved in synovitis, thus promoting the disease progression (18 19). In this study, telmisartan, as AT1R inhibitor, inhibited the AT1R expression and up-regulated the AT2R expression. Telmisartan promoted the proliferation and inhibited the apoptosis of ATDC5 cells and oxygen-glucose deprivation ATDC5 cells and improved the synthesis and secretion of collagen II in ATDC5 cells and oxygenglucose deprivation ATDC 5 cells by inhibiting AT1R and stimulating AT2R. The above findings were consistent with the functions of AT1R and AT2R in arthritis demonstrated in the present study.

\section{Conclusion}

Generally, Ang II suppressed the proliferation of oxygen-glucose deprivation ATDC5 cells by increasing AT1/2R expression and ratio of AT1R to AT2R. Down-regulation of AT1R and up-regulation of AT2R caused by telmisartan promoted the proliferation and inhibited the apoptosis of oxygen-glucose deprivation ATDC5 cells. Our findings revealed the underlying mechanism of AT1/2R expression for OA development, which contributes to exploration of effective therapeutic targets for OA.

\section{Reference}

1. Siebert S, Tsoukas A, Robertson J et al. Cytokines as Therapeutic Targets in Rheumatoid Arthritis and Other Inflammatory Diseases. Pharmacol Rev 2015; 67 (2): 280-309. doi: 10.1124/pr.114.009639.
2. Loeser RF, Goldring SR, Scanzello CR et al. Osteoarthritis: A disease of the joint as an organ. Arthritis Rheum 2012; 64 (6): 1697-707. doi: 10.1002/ art.34453.

3. Hunter DJ, Lo GH. The Management of Osteoarthritis: An Overview and Call to Appropriate Conservative Treatment. Med Clin NAm 2009; 93 (1): 127-143. doi: https://doi.org/10.1016/j.mcna.2008.07.009

4. Suri P, Katz JN, Rainville J et al. Vascular disease is associated with facet joint osteoarthritis. Osteoarthritis and Cartilage 2010; 18 (9): 1127-1132. doi: https://doi.org/10.1016/j.joca.2010.06.012.

5. Hochberg MC. New paradigms in the management of osteoarthritis patients with hypertension. Osteoarthritis Cartilage 2010; 18 (5): S1-S2. doi: https://doi.org/10.1016/j.joca.2010.04.001.

6. Felson DT, Zhang Y. An update on the epidemiology of knee and hip osteoarthritis with a view to prevention. Arthritis \& Rheumatism 1998; 41 (8): 13431355. doi: 10.1002/1529-0131 (199808)41:8<1343::AID-ART3>3.0.CO; 2-9.

7. AD T, G T, L H et al. Novel mechanisms of G-protein-coupled receptors functions: AT angiotensin receptor acts as a signaling hub and focal point of receptor cross-talk. Best practice \& research Clin Endocrinol Metab 2018; 32 (2): 69-82. doi: 10.1016/j.beem.2018.02.003.

8. CA M, A V, AA M et al. Direct angiotensin AT2 receptor stimulation using a novel AT2 receptor agonist, compound 21, evokes neuroprotection in conscious hypertensive rats. PloS one 2014; 9 (4): e95762. doi: 10.1371/ journal.pone.0095762.

9. Y W, M DB, HW L et al. Anti-fibrotic Potential of AT Receptor Agonists. Front Pharmacol 2017; 8:564. doi: 10.3389/fphar.2017.00564.

10. I D, Q A, T H. Proximal tubule angiotensin AT2 receptors mediate an anti-inflammatory response via interleukin-10: role in renoprotection in obese rats. Hypertension (Dallas, Tex : 1979) 2013; 61 (6): 1218-26. doi: 10.1161/ hypertensionaha.111.00422.

11. ID, W M, T H.Angiotensin AT2 receptor stimulation is anti-inflammatory in lipopolysaccharide-activated THP-1 macrophages via increased interleukin-10 production. Hypertens Res 2015; 38 (1): 21-92. doi: 10.1038/hr.2014.132.

12. AZ A, HA E-S, AS O et al. Patterns of angiotensin converting enzyme insertion/deletion gene polymorphism among an Egyptian cohort of patients with rheumatoid arthritis. Internat J Rheum Dis 2013; 16 (3): 284-290. doi: 10.1111/j.1756-185X.2012.01820.x.

13. Yingzhen, Wang, Jianqiang et al. The renin-angiotensin system in the synovium promotes periarticular osteopenia in a rat model of collagen-induced arthritis. Int Immunopharmacol 2018; 65: 550-558.

14. Liu Q, Tian J, Xu Y et al. Protective Effect of RA on Myocardial Infarction-Induced Cardiac Fibrosis via AT1R/p38 MAPK Pathway Signaling and Modulation of the ACE2/ACE Ratio. J Agricult Food Chem 2016; 64 (35): 6716-6722. doi: 10.1021/acs.jafc.6b03001.

15. MacKenzie A, Dunning L, Ferrell WR et al. Angiotensin II Type 1 receptor blockade protects endothelium-derived hyperpolarising factor-mediated relaxation in a rat model of monoarthritis. Life Sci 2013; 92 (23): 1131-1137. doi: https://doi.org/10.1016/j.lfs.2013.04.011.

16. Peng-fei $\mathbf{Z}$, Ji-xiang $\mathbf{S}$, Si-hang $\mathbf{T}$ et al. Advances in Research on $\mathrm{Hy}$ poxic Environment and Knee Osteoarthritis. World Latest Med Inform 2019; 19 (63): 114-116.

17. M H, C S, UM S et al. Small-molecule AT2 receptor agonists. Medicinal research reviews 2018; 38 (2): 602-624. doi: 10.1002/med.21449.

18. Y C, W W. Angiotensin II in inflammation, immunity and rheumatoid arthritis. Clin Exp Immunol 2015; 179 (2): 137-145. doi: 10.1111/cei.12467.

19. $\mathbf{D ~ W}, \mathbf{S} \mathbf{H}, \mathbf{J} Z$ et al. Angiotensin II type 2 receptor correlates with therapeutic effects of losartan in rats with adjuvant-induced arthritis. J Cell Mol Med 2013; 17 (12): 1577-87. doi: 10.1111/jcmm.12128.

Received April 2, 2020. Accepted May 15, 2020. 